\title{
LIQUID SCINTILLATION COUNTER CHARACTERIZATION, OPTIMIZATION AND BENZENE PURITY CORRECTION
}

\section{F. G. MCCORMAC}

The Queen's University of Belfast, School of Geosciences, Palaeoecology Centre Belfast BT7 1NN, Northern Ireland

\begin{abstract}
In liquid scintillation counting (LSC), small variations in benzene purity can cause ${ }^{14} \mathrm{C}$ pulse-height spectra to move with respect to the counting window. Thus, one must carefully monitor the purity of each benzene sample and apply corrections for spectral shifts. I describe here the techniques used at Queen's University Belfast for deriving correction factors for observed small variations in benzene purity. I also describe the methods used at our laboratory to fine-tune our Quantulus LS counters for high-precision dating. The tuning of the instruments minimizes the effect of fluctuations in gain that may occur during the long counting periods required for high-precision dating. Any remaining influences on efficiency owing to gain changes are corrected for, along with the purity correction, by continuous monitoring of the spectrum produced by the external source.
\end{abstract}

\section{INTRODUCTION}

The accurate and precise measurement of the weak $\beta^{-}$radiation used in ${ }^{14} \mathrm{C}$ dating requires that carbon from the sample be converted, with a high efficiency, into a medium from which low-level radiation detection is possible, e.g., benzene, in the case of liquid scintillation counters (LSCs) and acetylene, methane or carbon dioxide, in the case of gas counters. Counters (either LS or gas proportional) suitable for the accurate and precise measurement of the weak $\beta^{-}$radiation are specially designed to give high-detection efficiency, long-term stability and low background contributions. Despite great technical advances in LSC design, errors at the counting stage of the dating process can still arise from impurities in the synthesized benzene. Using a commercially available chromium-activated cracking catalyst, Switsur and Waterhouse (1989) analyzed the impurities in benzene produced by cyclotrimerization of acetylene. Although they showed the levels of impurities (principally, ethylbenzene, toluene, acetone, (1-methylpropyl)benzene and isopropylbenzene) to be small ( 86 ppm or less, see also Polach, Gower \& Fraser 1973; Coleman et al. 1973; Tamers 1975; Witkin et al. 1991), this study shows that, even at such low levels, impurities can contribute to dating errors, particularly for older material. Impurities affect the measured activity of a sample by causing shifts in the resultant energy spectrum, thereby reducing the counting efficiency within a given counting window. Birks (1970) showed that the concentration of dissolved oxygen also affects the degree of quenching. Thus, vials filled at different ambient temperatures and pressures are quenched by different amounts, even if the benzene has identical impurity levels at the outset. It is important that spectral shifts caused by all forms of quenching are corrected for in high-precision dating. Described here is a method for the monitoring and correction of spectral shifts caused by variable quenching of benzene containing low levels of impurities. Because small gain changes in LSCs can also affect the efficiency during the long counting periods required for high-precision measurements, we quasi-continuously monitor the external source spectrum and correct for gain changes along with the purity correction (i.e., purity variation causes a primary spectral shift and gain changes result in spectral variation about the offset level).

\section{LIQUID SCINTILLATION COUNTERS}

The LSCs used at Queen's University, Belfast are LKB Quantulus 1220s. These instruments have been modified by the manufacturer so that the automatic gain control on the left and right phototubes can be overridden and the high voltages can be set independently on either phototube. 
The modification also provides for a single manual high-voltage adjustment on the guard phototubes. The principle behind the automatic gain control is that light-emitting diodes (LEDs) in juxtaposition to the phototubes flash on and off at approximately $60 \mathrm{~Hz}$. Since the LEDs are carefully controlled to emit a constant light flux, the output response of the phototubes to the light from the LEDs should be constant. A feedback circuit to the high-voltage controller adjusts the high voltage to maintain a constant output from the phototubes in response to the LED light. Whereas the feedback circuitry represents a great step in technology and is labor-saving for routine ${ }^{14} \mathrm{C}$ dating, it is not used in high-precision dating. Instead, the high voltages are set manually, allowing for improved optimization and eliminating the need for the LEDs, which can thus be turned off. This reduces the exposure of the photocathodes to light, and may help prolong the life and stability of the phototubes.

Independent manual control of the high voltages requires that spectra from the left and right tubes be determined independently and the high voltages adjusted so that the two match exactly. The spectra may not be optimally matched in the instrument as supplied, and the flexibility introduced by manual control allows for improved optimization. Optimization of the left and right tubes can be performed in several ways. We generate two graphs (one for each phototube), which give the internal spectral quench parameter, SQP(I) (this is the channel position of the center of gravity of the ${ }^{14} \mathrm{C} \beta^{-}$spectrum from a high activity $(1692 \mathrm{dpm} / \mathrm{g}){ }^{14} \mathrm{C}$ benzene sample), as a function of high voltage (details of selection of left and right spectra are given in the LKB service manual under Section F, spectra adjustment). By recording SQP(I) as a function of high voltage for each tube (see Fig. 1), two linear graphs are obtained and a regression line is fitted to each. From this, high voltages are determined for identical SQP(I) values, and the spectra can be matched. LKB set SQP(I) to $407 \pm 1$ for both tubes.

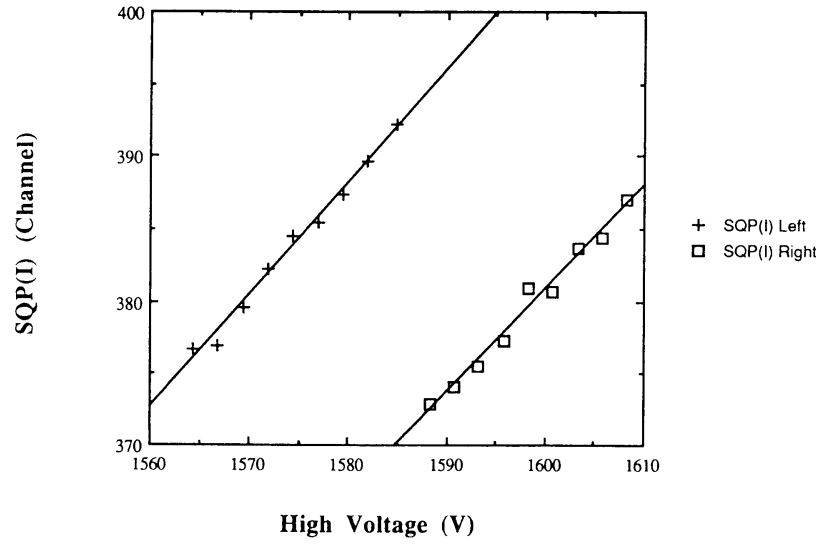

Fig. 1. Graph of SQP(I) vs. high voltage for both left and right phototubes independently

It is important that $\mathrm{SQP}(\mathrm{I})$ is used to match the left and right phototube spectra, and not SQP(E). $\mathrm{SQP}(\mathrm{E})$ is the channel above which $1 \%$ of the observed counts are obtained when the $10 \mu \mathrm{g}{ }^{226} \mathrm{Ra}$ $(3.7 \mathrm{KBq})$ external source is presented to the base of the vial. Since the dynamic response of the tubes as a function of $\beta^{-}$energy are not identical and the Compton continuum produced by the ${ }^{226} \mathrm{Ra}$ source is quite dissimilar from the ${ }^{14} \mathrm{C}$ spectrum, significant offsets between left and right external spectra may occur at the higher energy levels, even though the ${ }^{14} \mathrm{C}$ spectra are well matched. 


\section{BALANCE-POINT COUNTING}

Surface water used in our benzene synthesis (Barker 1953) contains ${ }^{3} \mathrm{H}$, which is incorporated into the synthesized benzene (Pearson 1983). Because of overlap of the ${ }^{3} \mathrm{H}$ (maximum $\beta^{-}$energy of $0.018 \mathrm{MeV}$ ) and ${ }^{14} \mathrm{C}$ (maximum $\beta^{-}$energy of $0.156 \mathrm{MeV}$ ) energy spectra, it is not possible to use all $\beta^{-}$decays within the energy range emitted by ${ }^{14} \mathrm{C}$ for ${ }^{14} \mathrm{C}$ dating. Instead, a lower energy discriminator is defined, so that the contribution of ${ }^{3} \mathrm{H}$ to the final ${ }^{14} \mathrm{C}$ counts is minimal $(<1 \%$ of the total ${ }^{3} \mathrm{H}$, see below).

The simplest measure of instrument performance is provided by the Figure of Merit $\left(E^{2} / B\right)$, where $E$ is the observed counting efficiency, and B, the background count rate. If the goal, as is often the case in radioisotope measurement, were to maximize the Figure of Merit, then all disintegrations detected with energy above the lower-discriminator up to the maximum energy of $\beta^{-}$emission for ${ }^{14} \mathrm{C}$ could be used in determining the activity of a sample. However, accuracy and precision require long-term instrument stability; thus, it is considered judicious to sacrifice counts at the higher $\beta^{-}$ energies to operate the counter at 'balance-point'. Operating at 'balance-point' requires that an upper-level discriminator be defined, such that the counts recorded in a sample at the lower-discriminator channel are approximately equal to the counts recorded at the upper-discriminator channel. During a counting period, the effect of small fluctuations in system gain, that result in ${ }^{14} \mathrm{C}$ spectral shifts around the balance-point position are then minimized. As the spectrum moves within the counting window, counts that are lost at one side of the spectrum are gained at the other, resulting in only slightly reduced total counting efficiency. It is important to note that balance-point operation does not provide a constant counting efficiency, but only reduces the effect that small system gain changes have on the efficiency (Pearson 1979).

Any external factor that causes a change in the pulse-height spectrum will result in a ${ }^{14} \mathrm{C}$ spectrum moving with respect to the balance-point position. Thus, it is necessary to normalize all spectra by applying an appropriate correction to the counts obtained in the ${ }^{14} \mathrm{C}$ window, thereby simulating a constant efficiency. (I describe below corrections for spectral shifts caused by quenching and other factors (such as photomultiplier gain changes) that affect the pulse-height spectrum and shift the mean position of the spectrum in the counting window.)

\section{TRITIUM INTERFERENCE}

${ }^{3} \mathrm{H}$ is incorporated into the benzene primarily by reacting water with lithium carbide to generate acetylene. The acetylene is subsequently trimerized on a chromium catalyst to produce benzene by a method similar to that described by Noakes, Kim and Stipp (1965). Although considerable efforts have been made to locate and use a water source with a low ${ }^{3} \mathrm{H}$ content, some ${ }^{3} \mathrm{H}$ is still present in the final benzene (Pearson 1983). Because the high energy end of the ${ }^{3} \mathrm{H}$ spectrum overlaps the low energy end of the ${ }^{14} \mathrm{C}$ spectrum, a contribution from ${ }^{3} \mathrm{H}$ exists in the final ${ }^{14} \mathrm{C}$ count rate. To minimize this and retain as much as possible of the ${ }^{14} \mathrm{C} \beta^{-}$counts, the lower energy discriminator is set, so that only $1 \%$ of the ${ }^{3} \mathrm{H}$ is included in the final ${ }^{14} \mathrm{C}$ spectrum. The $1 \%$ level is determined by obtaining the spectrum of a tritiated benzene sample and determining the energy level (channel), above which only $1 \%$ of the ${ }^{3} \mathrm{H}$ counts is recorded. About $8 \%$ of ${ }^{14} \mathrm{C} \beta^{-}$decays are lost by positioning the lower discriminator at this level (see Pearson 1979).

\section{DETERMINATION OF UPPER DISCRIMINATOR FOR BALANCE POINT OPERATION}

To operate at balance point, one must first determine the lower energy discriminator level as described above. Then, using a high-activity ${ }^{14} \mathrm{C}$ benzene sample $\left(1.692 \times 10^{3} \mathrm{dpm} / \mathrm{g}\right.$ in our case), an energy spectrum is recorded for $100 \mathrm{~min}$. The spectrum, which is in counts per minute (cpm) 
as a function of log (energy), is then used to determine the upper discriminator level by locating the channel where the count rate equals that in the lower discriminator channel. The count rate at the lower discriminator channel is taken as the mean of five channels on either side of the lower discriminator. Thus, the upper discriminator channel is determined by taking an 11-point running mean of the counts in the higher energy channels and finding the channel for which the running mean value at the higher energy equals that at the lower discriminator. For our Quantulus counters, the channel separation between lower and upper discriminators (i.e., ${ }^{14} \mathrm{C}$ counting window) is about 275 channels.

\section{DETERMINATION OF THE CORRECTION FACTOR}

When both left and right phototubes have been matched, and the upper and lower level discriminators have been determined, it is then possible to proceed to determine the normalization constants to simulate a constant efficiency. A parameter that accurately monitors the position of the spectra in the Quantulus counters is the SQP(E) value (i.e., the channel above which $1 \%$ of the observed counts from the Compton continuum is obtained when the $10 \mu \mathrm{g}{ }^{226} \mathrm{Ra}(3.7 \mathrm{KBq})$ external source is presented to the base of the vial). We monitor SQP(E) for $10 \%$ of the sample counting time (i.e., $10 \mathrm{~min}$ for every 100 -min counting period). By determining the change in efficiency as a function of SQP(E) for a fixed counting window, and monitoring SQP(E) for each sample, one can correct for efficiency variations caused by intersample quench variability (i.e., a quench correction curve). Because the purity of the benzene we synthesize is very high, the quench corrections are small, and thus, we cannot determine a quench correction curve with sufficient accuracy from a set of quenched standards. Thus, we simulate the standards by slightly altering the high voltages on the phototubes (during setup) and recording the SQP(E) values for high-activity spectrophotometric benzene as a function of high-voltage (i.e., we alter the high voltage by $\pm 5 \mathrm{~V}$ to simulate a gain change). The high voltage on both left and right tubes is adjusted by $c a .1 \mathrm{~V}$ at a time (the exact voltage adjustment for left and right tubes is determined from the regression line in Figure 1, to ensure that the spectra remain matched (i.e., have equal SQP(I) values) as the high voltages are varied).

The ${ }^{14} \mathrm{C}$ spectra and associated $\mathrm{SPQ}(\mathrm{E})$ values recorded at the range of high voltages are stored for subsequent computer analysis. A computer program that passes a window, identical in width to the balance-point window, over each of the spectra and sums the counts within the window for each channel step, is used to determine the position of maximum efficiency for each spectrum. The lower-discriminator channel at maximum efficiency is noted for each spectrum. A graph is then plotted of SQP(E) as a function of lower discriminator channel (Fig. 2).

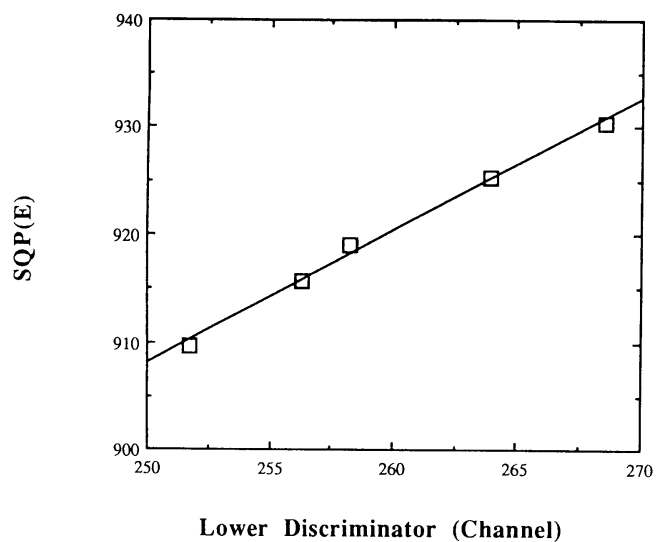

Fig. 2. Lower discriminator channel as a function of $S Q P(E)$ 


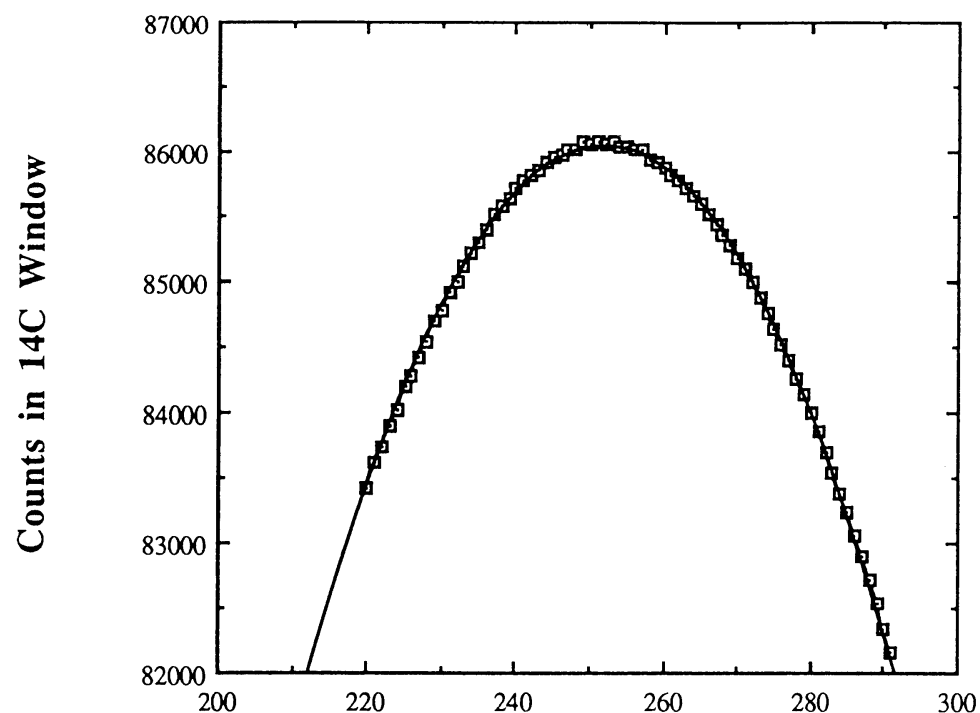

Lower Discriminator Channel
Fig. 3. Counts within ${ }^{14} \mathrm{C}$ counting window as a function of lower discriminator channel. $\square=$ individual data points; a parabolic curve is fitted to the data.

The high voltages are set at their operating levels and a ${ }^{14} \mathrm{C}$ spectrum obtained from the highactivity standard. The spectrum is input to the computer program, which steps the counting window, one channel at a time, through the spectrum, and a plot of total counts within the window as a function of lower discriminator channel is generated (Fig. 3). The lower discriminator channel, which forms the abscissa of Figure 3, is converted to SQP(E), with the results obtained in Figure 2 giving a graph of counts $v$ s. SQP(E) (Fig. 4). An orthonormalized Legendre polynomial of order 2 (i.e., a parabola) is fitted to the data in Figure 4, and the peak of the parabola is determined in terms of SPQ(E)

$$
\mathrm{SQP}(\mathrm{E}) \mathrm{bal}=\frac{-\mathrm{b}}{2 \mathrm{a}}
$$

where $a$ and $b$ are the coefficients of the $x^{2}$ and $x$ terms in the polynomial, respectively. The total counts at this position represent the maximum counting efficiency of the matched balance-point spectrum. To determine a correction for $\mathrm{SQP}(\mathrm{E})$ variations in sample benzene, we use the following formula

$$
\text { Correction factor }=\frac{\mathrm{aSQP}(\mathrm{E})^{2} \mathrm{bal}+\mathrm{bSQP}(\mathrm{E}) \mathrm{bal}+\mathrm{c}}{\mathrm{aSQP}(\mathrm{E})^{2} \text { sample }+\mathrm{bSQP}(\mathrm{E}) \text { sample }+\mathrm{c}}
$$

where $a, b$ and $c$ are the coefficients determined from the Legendre polynomial fit, $\operatorname{SQP}(E) b a l$ is the external spectral quench parameter for a balance point spectrum and SQP(E)sample is the external spectral quench parameter for a sample spectrum. The $\mathrm{cpm} / \mathrm{g}$ is multiplied by the correction factor after the background has been subtracted. Figure 5 shows correction factors as a function of SQP(E) for one of our counters. This curve should be determined for each individual counter and needs to be reevaluated about every three months or after any change is made to the operating voltages or settings. 


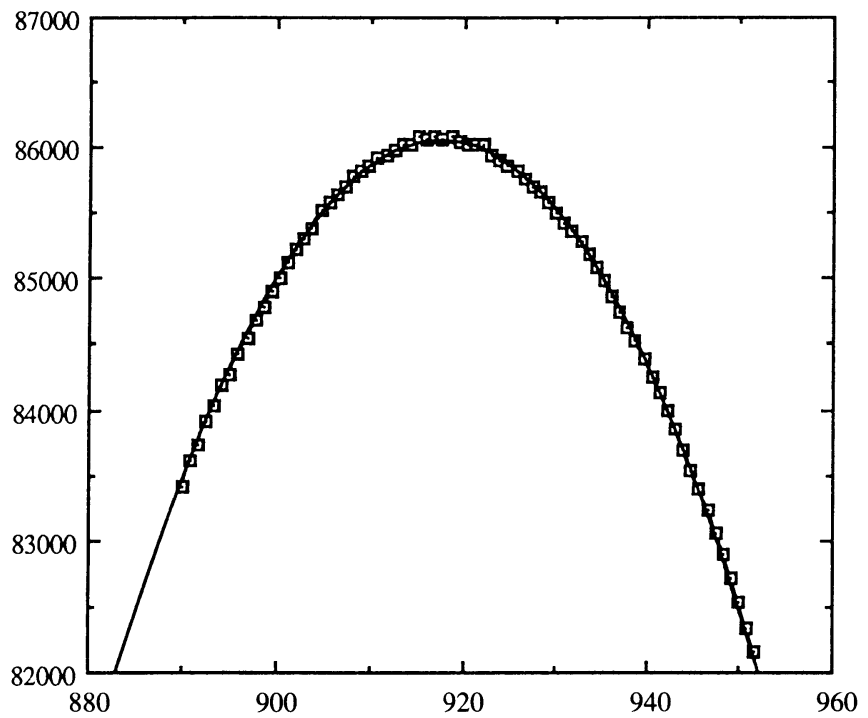

Fig. 4. Counts within ${ }^{14} \mathrm{C}$ counting window as a function of SQP(E). See Figure 3, for sym-

$\operatorname{SQP}(E)$

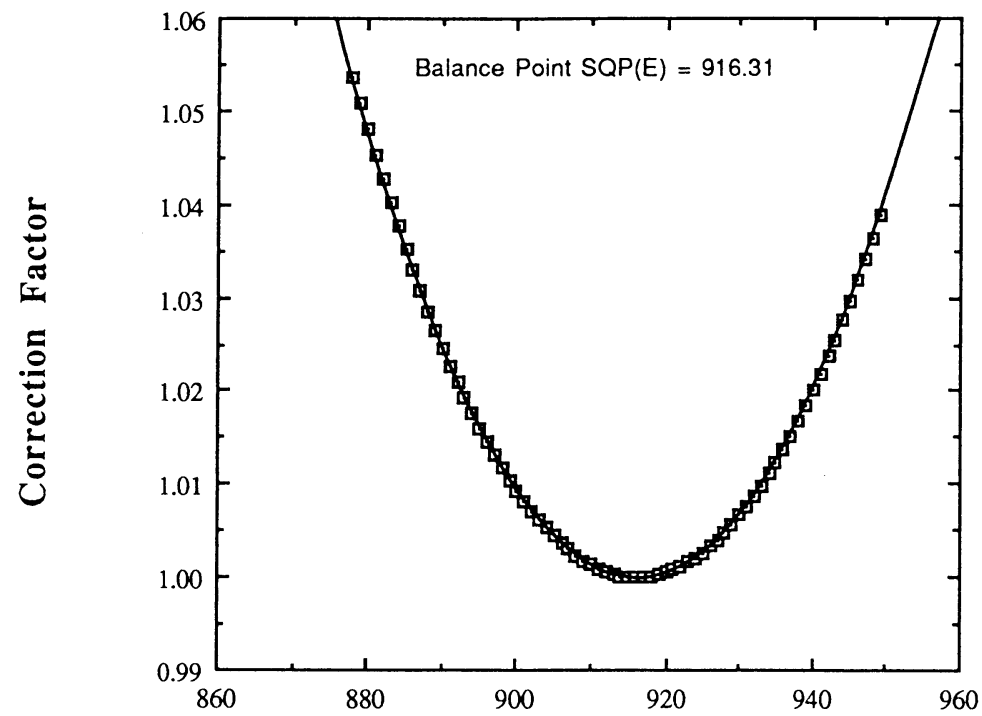

Fig. 5. Correction factor by which counts within counting window should be multiplied as SQP(E) varies

\section{SQP(E)}

\section{EFFECT OF SPECTRAL SHIFTS ON AGE DETERMINATION}

The SQP(E) correction factor described above can be expressed as an error on the ${ }^{14} \mathrm{C}$ age. Figure 6 gives the age corrections (i.e., the age by which the determined date would be too old if no correction was applied) for each channel difference in SQP(E) from that determined with the active standard used during setup. As can be seen in Figure 6, very small purity changes result in a negligible error on the final date. However, significant biases are introduced at $>2$ channels shift 


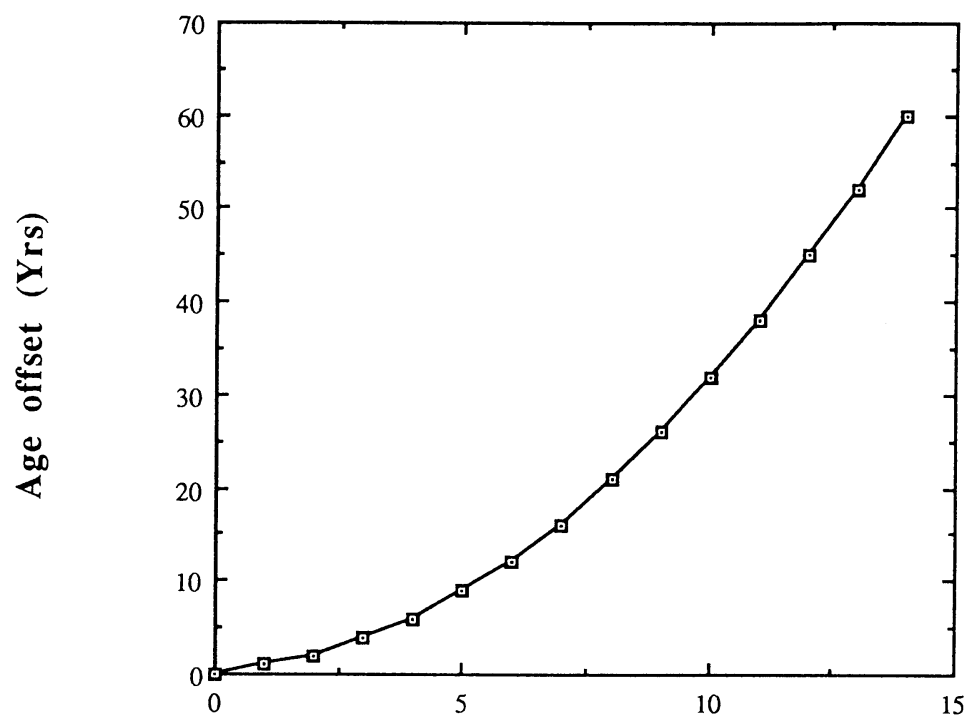

SQP(E) shift from setup position
Fig. 6. Error in ${ }^{14} \mathrm{C}$ age as a function of SQP(E) if purity and gain variations are not corrected for

in $\operatorname{SQP}(E)$. We have confirmed the results obtained in Figure 6 by dating a highly quenched benzene sample derived from charcoal obtained at an archaeological site in north Antrim, Northern Ireland. The date for the sample was $4051 \pm 24 \mathrm{BP}$. Had no correction for quenching been applied to the counts from this sample, the resultant date would have been $4112 \pm 24 \mathrm{BP}$. The sample was vacuum-distilled to remove impurities and recounted. The new date determined was $4062 \pm 21 \mathrm{BP}$, with only a small correction needed for quenching. Thus, within the error limits, the correction factor had satisfactorily accounted for the quenching.

Because the background is not uniform with energy, when a ${ }^{14} \mathrm{C}$ spectrum shifts with respect to the counting window, it is subject to a different background contribution. This variation in background can significantly affect the result when dating very old samples, at $c a .50 \mathrm{ka}$. To investigate the effect of purity changes on background contribution, we used an accumulated background spectrum to compute the total counts within the counting window as a function of $\mathrm{SQP}(\mathrm{E})$ (the method was identical to that described above for the ${ }^{14} \mathrm{C}$ spectrum). By determining the total counts within the background counting window at balance point, and normalizing to this value, we determined a multiplier for the background as a function of $\operatorname{SQP}(E)$ (Fig. 7). For material in the 40 to $50 \mathrm{ka}$ range, application of the background correction can alter the date by as much as $1 \mathrm{ka}$ depending on the background level. For more recent samples (e.g. 0-10 ka), the error is again background- level-dependent, but usually $<2$ a.

\section{CONCLUSIONS}

I have presented methods used to characterize and optimize LSCs at The Queen's University of Belfast. Although the description relates to LKB Quantulus instruments and terminology, the principles outlined are identical for other LSC types. If high-precision dating is to be attempted, it is vital that the LSC is:

1. Operated at balance-point for long-term stability, low background and optimal efficiency (within the constraints of balance-point counting) 


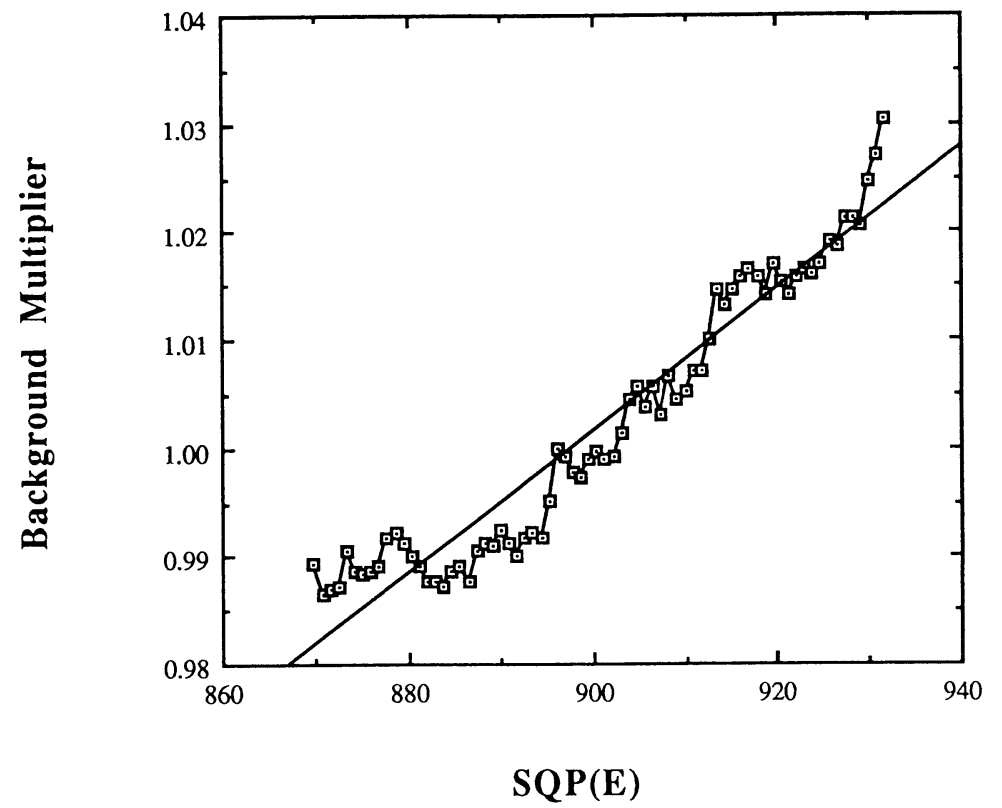

Fig. 7. Background multiplier as a function of SQP(E). The straight line represents a linear regression fit to the background multiplier. The data points are the individual background multiplier values determined by normalizing the counts within the counting window at a particular $\operatorname{SQP}(E)$ value to the counts in the counting window at balance-point.

2. Characterized in terms of the spectral shifts of each phototube as a function of high-voltage, so that the spectra from left and right phototubes are matched and remain so

3. Characterized to correct for spectral shifts resulting from variation in benzene purity due to 1) alkylbenzenes introduced during the trimerization process, 2) varying dissolved oxygen levels in the benzene due to ambient temperature and pressure variations during vial filling, and 3) instrument gain changes.

It is of utmost importance in high-precision dating to apply corrections for spectral shifts resulting from variable levels of quenching in synthesized benzene and for instrument gain changes. Without these corrections, errors in excess of 60 a on material from the past 10 millennia, and in excess of $1 \mathrm{ka}$ on material in the 40 to $50 \mathrm{ka}$ range (depending upon background levels) can occur.

\section{ACKNOWLEDGMENTS}

The author wishes to acknowledge useful discussions with G. W. Pearson, R. M. Kalin, M. G. L. Baillie and J. R. Pilcher. Thanks are also due S. Hoper and F. Sharpe for technical assistance. 


\section{REFERENCES}

Barker, H. 1953 Radiocarbon dating: Large scale preparation of acetylene from organic material. Nature 172: 631-632.

Birks, J. B. 1970 Physics of the liquid scintillation process. In Bransome, E. D., ed., The Current Status of Liquid Scintillation Counting. New York, Grune and Shatton.

Coleman, D. D., Liu, C. L., Dickerson, D. R. and Frost, R. R. 1973 Improvement in trimerisation of acetylene to benzene for radiocarbon dating with a commercially available vanadium oxide catalyst. In Rafter, T. A. and Grant-Taylor, T., eds., Proceedings of the 8th International ${ }^{14} \mathrm{C}$ Conference. Wellington, Royal Society of New Zealand 1: 158-170.

Noakes, J. E., Kim, S. M. and Stipp, J. J. 1965 Chemical and counting advances in liquid scintillation age dating. In Olsson, E. A. and Chatters, R. M., eds., Proceedings of the 6th International Conference on Radiocarbon and Tritium Dating. Clearinghouse for Federal Scientific and Technical Information, Washington, DC: 68-92.

Polach, H., Gower, J. and Fraser, I., 1973 Synthesis of high purity benzene for radiocarbon dating. In Rafter, T. A. and Grant-Taylor, T., eds., Proceedings of the 8 th International ${ }^{14} \mathrm{C}$ Conference. Wellington, Royal Society of New Zealand, 1: B36- B49.

Pearson, G. W. 1979 Precise ${ }^{14} \mathrm{C}$ measurement by liquid scintillation counting. Radiocarbon 21(1): 1-21.

1983 The development of high precision ${ }^{14} \mathrm{C}$ measurement and its application to archaeological time-scale problems. Ph.D. thesis, The Queen's University Belfast.

Switsur, R. and Waterhouse, J. S. 1989 Benzene purity in radiocarbon dating samples. In Long, $\mathrm{A}$. and $\mathrm{Kra}$, R. S., eds., Proceedings of the 13 th International ${ }^{14} \mathrm{C}$ Conference. Radiocarbon 31(3): 260-263.

Tamers, M. A. 1975 Chemical yield optimisation of the benzene synthesis for radiocarbon dating. International Journal of Applied Radiation Isotopes 26: 676682.

Witkin, D., Rigali, M. J., Kalin, R. M., Nagy, B. and Long, A. 1991 Impurities arising during benzene synthesis from acetylene on vanadium and chromium catalysts. Radiocarbon 33(2): 261-262. Abstract. 